\title{
Manutenção do aleitamento materno de recém-nascidos pré-termo: revisão integrativa da literatura
}

\author{
Maintaining the breastfeeding of preterm newborns: an integrative literature review
}

Continuidad de la lactancia materna de recién nacidos prematuros: revisión integrativa de la literatura

Andressa Peripolli Rodrigues ${ }^{1}$, Elaine Lutz Martins², Tatiane Correa Trojahn ${ }^{3}$, Stela Maris de Mello Padoin ${ }^{4}$, Cristiane Cardoso de Paula ${ }^{5}$, Caroline Sissy Tronco ${ }^{6}$

\footnotetext{
${ }^{1}$ Enfermeira. Discente do Programa de Pós-Graduação em Enfermagem da Universidade Federal de Santa Maria (UFSM), nível Mestrado. Santa Maria, RS, Brasil. E-mail: andressaufsm@hotmail.com.

2 Enfermeira. Santa Maria, RS, Brasil. E-mail: elainelutzmartins@yahoo.com.br.

${ }^{3}$ Enfermeira. Discente do Programa de Pós-Graduação em Enfermagem da UFSM, nível Mestrado. Enfermeira do Hospital Universitário de Santa Maria/UFSM. Santa Maria, RS, Brasil. E-mail: tatitrojahn@yahoo.com.br.

${ }^{4}$ Enfermeira, Doutora em Enfermagem. Professora Adjunta da UFSM. Santa Maria, RS, Brasil. E-mail: stelamaris padoin@hotmail.com.

${ }^{5}$ Enfermeira, Doutora em Enfermagem. Professora Adjunta da UFSM. Santa Maria, RS, Brasil. E-mail: cris depaula1@hotmail.com.

${ }^{6}$ Enfermeira. Discente do Programa de Pós-Graduação em Enfermagem da UFSM, nível Mestrado. Enfermeira do Hospital Universitário de Santa Maria/UFSM. Santa Maria, RS, Brasil. E-mail: carolinetronco@hotmail.com.
}

\section{RESUMO}

A presente pesquisa trata-se de estudo de revisão integrativa com objetivo de avaliar as evidências disponíveis sobre fatores associados à manutenção do aleitamento materno de recém-nascidos pré-termo. Desenvolvido nas bases de dados MEDLINE e LILACS, com os descritores "aleitamento materno" e "prematuro". A amostra incluiu vinte estudos com nível de evidência 2 e 4, que apontaram fatores facilitadores e dificultadores associados à manutenção do aleitamento materno. São necessárias ações multiprofissionais no acompanhamento de saúde com estratégias educativas, de promoção do vínculo mãe-bebê e acolhimento da família. Deve-se ter especial atenção às condições sociais, econômicas e culturais da família, bem como às demandas clínicas dos recém-nascidos pré-termo para a manutenção do aleitamento.

Descritores: Aleitamento Materno; Enfermagem; Prematuro; Desmame.

\section{ABSTRACT}

This integrative review was performed with the objective to evaluate the available evidence regarding the factors associated with maintaining the breastfeeding of preterm newborns. The search was performed on the MEDLINE and LILACS databases using the descriptors "breast feeding" and "infant, premature". The sample included twenty studies with an evidence level of 2 and 4, which pointed out facilitating and hindering aspects associated with maintaining breastfeeding. There is a need for multidisciplinary interventions in the health follow-up program, including educational strategies that promote mother-baby attachment and family welcoming. Special care should be given to the social, economical and cultural conditions of the family, as well as the clinical demands of the preterm newborns to maintain breastfeeding.

Descriptors: Breast Feeding; Nursing; Infant, Premature; Weaning.

\section{RESUMEN}

Estudio de revisión integrativa con el objetivo de evaluar las evidencias disponibles sobre factores asociados a la continuidad de la lactancia materna de recién nacidos prematuros. Desarrollado con las bases de datos MEDLINE y LILACS, con los descriptores "Lactancia Materna" and y "Prematuro". La muestra incluyó 20 estudios con nivel de evidencia 2 y 4, que expresaron factores facilitadores y limitantes asociados a la continuidad de la lactancia materna. Son necesarias acciones de tipo multiprofesional en el seguimiento de salud con estrategias educativas de promoción del vínculo mamá-bebé y apoyo familiar. Debe ponerse especial atención en las condiciones sociales, económicas y culturales de la familia, así como en las demandas clínicas de los recién nacidos prematuros para la continuidad de la lactancia.

Descriptores: Lactancia Materna; Enfermería; Prematuro; Destete. 


\section{INTRODUÇÃO}

O leite humano apresenta composição balanceada de nutrientes essenciais para o crescimento e desenvolvimento do recém-nascido (RN), sendo adaptado ao metabolismo da criança. A Organização Mundial da Saúde (OMS) recomenda o leite humano durante os primeiros seis meses de vida, pois é suficiente para suprir as necessidades nutricionais do bebê, quando em aleitamento materno exclusivo (AME). Indica que após os seis meses, se inicie gradativamente a alimentação complementar mantendo a amamentação até pelo menos os dois anos de idade ${ }^{(1-2)}$.

O leite materno é a melhor escolha para o recémnascido pré-termo (RNPT), criança nascida antes de 37 semanas de gestação. $O$ aleitamento materno (AM) supre suas necessidades e deve ser o alimento de escolha, pois contém mais proteínas, lipídeos e calorias, se comparado ao leite de mães de recém-nascido a termo ${ }^{(1-3)}$.

Devido às condições clínicas e ao ambiente hospitalar, amamentar o RNPT é difícil e desafiador. As mães podem se sentir pouco confortáveis em lidar com esses bebês pequenos e clinicamente frágeis, o que as fazem concluir que são incapazes de amamentá-los. Assim, diante das dificuldades no cotidiano das mães e da vivência da internação do seu filho, os profissionais de saúde devem garantir uma assistência adequada e benéfica para a mulher e para seu bebê( ${ }^{(3)}$.

A prevalência do AME em RNPT mostra-se aquém do valor recomendado pela OMS. Um estudo brasileiro constatou a prevalência de AME de $36 \%$ na alta hospitalar, aumentando para $54,7 \%$ após a implantação da Iniciativa Hospital Amigo da Criança(4). Outro estudo constatou uma prevalência de AME na alta hospitalar de $58,3 \%$ e de $22,2 \%$ aos seis meses de vida ${ }^{(5)}$.

As crianças que ficam internadas na Unidade de Terapia Intensiva Neonatal (UTIN) apresentam dificuldades no início e na manutenção do $A M$, vivenciadas também por suas mães. É necessário promover e apoiar a alimentação adequada, fornecendo informações e suporte para o manejo do AM, almejando a sua efetivação ${ }^{(3,6-7)}$.

Sendo assim, o objetivo deste estudo foi avaliar as evidências sobre os fatores associados à manutenção do AM em RNPT.
Estudo de revisão integrativa da literatura, com a finalidade de reunir e sintetizar resultados de pesquisas sobre um delimitado tema ou questão de maneira sistemática e ordenada. Para a realização da revisão seguiu-se as etapas: identificação do tema, seleção da questão de pesquisa e definição dos objetivos, estabelecimento de critérios de inclusão e exclusão, seleção dos estudos, estabelecimento das informações a serem extraídas dos artigos, avaliação das evidências e análise (categorização), discussão e apresentação da síntese do conhecimento evidenciado nos artigos analisados ${ }^{(8)}$.

Para orientar este estudo, a questão de pesquisa formulada foi: Quais os fatores associados à manutenção do AM em RNPT? A busca bibliográfica foi desenvolvida nas bases de dados eletrônicas Medical Literature Analysis and Retrieval System Online (MEDLINE) e Literatura Latino-Americana e do Caribe em Ciências da Saúde (LILACS), utilizando os descritores "aleitamento materno" and "prematuro".

O levantamento dos estudos ocorreu em março de 2012. Para selecioná-los, os critérios de inclusão foram: artigos de pesquisas com nível de evidência de um a quatro na classificação em sete níveis ${ }^{(9)}$, disponiveis online na íntegra; em inglês, português ou espanhol; e no recorte temporal de 1999 até 2011, pois o ponto inicial está sustentado na implantação da Política de Atenção Humanizada ao Recém-Nascido de Baixo Peso-Método Canguru(1). Os critérios de exclusão foram: artigos sem resumo na base de dados ou incompletos.

Conforme mostra a Figura 1, foram inicialmente encontrados 532 estudos, dos quais 20 atendiam aos critérios de inclusão.

\section{MÉTODOS}


Figura 1: Estrutura do desenvolvimento do estudo de revisão. LILACS, MEDLINE, 2012.

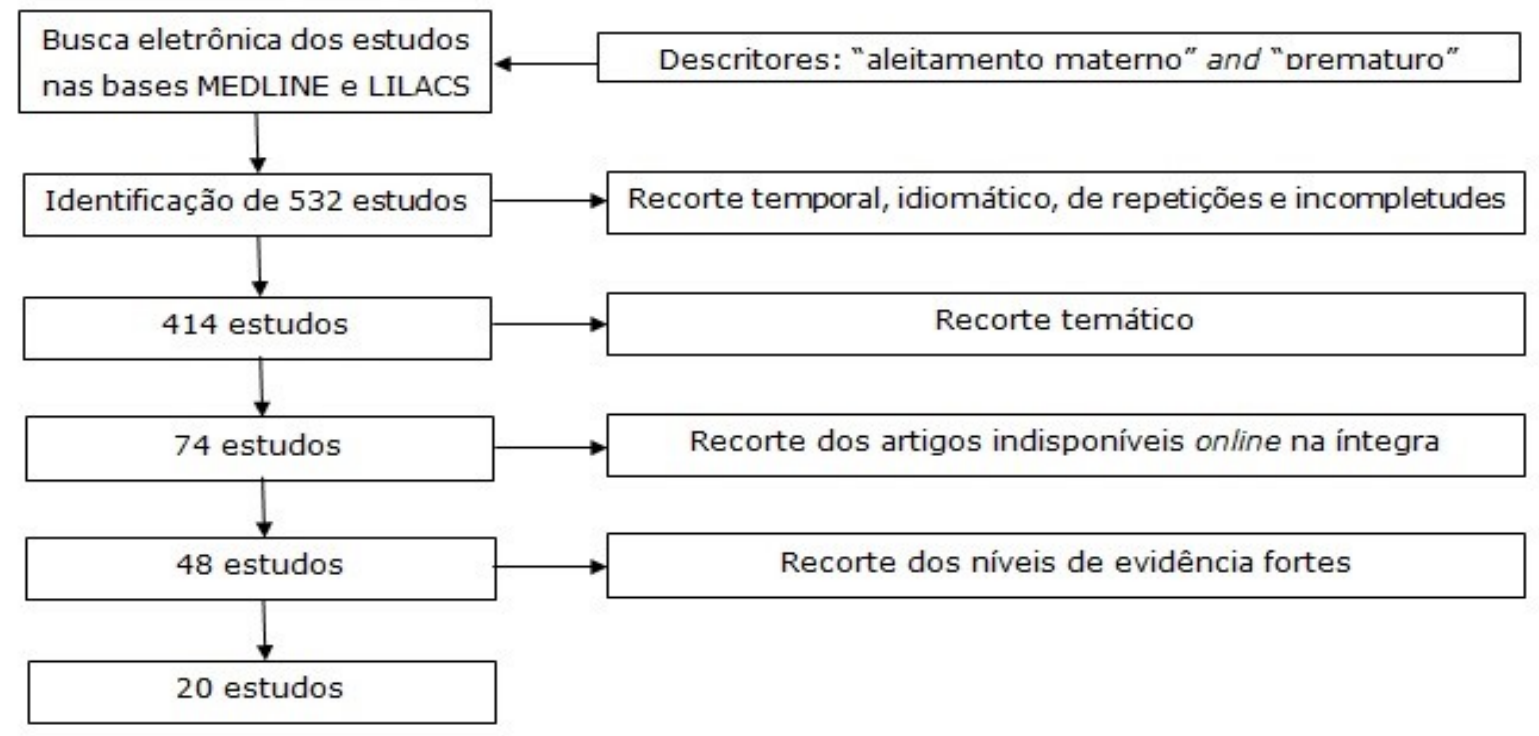

Após leitura exaustiva dos estudos selecionados, foi preenchido um instrumento contendo: referência, país onde o estudo foi realizado, subárea do conhecimento, objetivo e metodologia do estudo, nível de evidência e principais resultados do estudo.

Para minimizar possível viés de aferição dos estudos (erro de interpretação dos resultados e do delineamento), dois pesquisadores realizaram a leitura dos artigos e preenchimento do instrumento de forma independente, os quais posteriormente foram comparados. Não ocorreram divergências em relação à avaliação das publicações.

Os estudos foram classificados de acordo com os sete níveis de evidências descritos por Melnyk e FineoutOverholt $^{(9)}$.

\section{RESULTADOS}

A maior parte dos estudos foi publicada nos anos de 2011 (20\%) e 2007 (20\%), seguido do ano de 2001 (15\%). O Brasil (15\%) e a Suécia (15\%) concentraram a maior parte das publicações, seguidos da França, Estados Unidos da América, Israel e Itália (10\% cada). Quanto às subáreas de conhecimento, a maioria pertencia a Multiprofissional (40\%), Medicina (40\%) e Enfermagem (20\%). Referente ao delineamento dos estudos, nove (50\%) eram ensaio clínico randomizado, sete (35\%) coorte e quatro (15\%) caso controle. Segundo a classificação do nível de evidência ${ }^{(9)}$ constatou-se: nove estudos com nível de evidência $2^{(10-18)}$ e 11 estudos com nível $4^{(19-29)}$. O
Quadro 1 apresenta os estudos analisados, classificados por referência, objetivo, delineamento e principais resultados (Quadro 1). 
Quadro 1: Corpus da revisão integrativa. LILACS, MEDLINE. 2012.

\begin{tabular}{|c|c|c|c|}
\hline Referência & Objetivo & Delineamento & Principais Resultados \\
\hline $\begin{array}{l}\text { Hurst NM, Meier PP, Engstrom JL, } \\
\text { Myatt A. Mothers performing in-home } \\
\text { measurement of milk intake during } \\
\text { breastfeeding of their preterm } \\
\text { infants: maternal reactions and } \\
\text { feeding outcomes }{ }^{(10)}\end{array}$ & $\begin{array}{l}\text { Determinar as percepções de mães } \\
\text { ao usar teste de pesos em casa para } \\
\text { manejo da amamentação no } \\
\text { primeiro mês de alta da UTIN. }\end{array}$ & $\begin{array}{l}\text { ECR. n=31 mães de RNPT (amostra de conveniência) estratificado } \\
\text { por idade gestacional ( } 31 \text {-33 semanas, } 34-36 \text { semanas) } \\
\text { GE= medição da ingestão de leite } \\
\text { GC= não realizar medição } \\
\text { Variáveis: experiência de amamentação anterior; volume diário } \\
\text { de leite materno durante a UTIN; a frequência de mamada antes } \\
\text { da alta da UTIN; peso da criança na alta hospitalar e } 1,2 \text { e } 4 \\
\text { semanas; número de mamadas diárias, frequência, volume e tipo } \\
\text { de complemento e suplemento utilizado e quantidade de vezes } \\
\text { urinada. }\end{array}$ & $\begin{array}{l}\text { As mães que iniciaram a ordenha na UTIN } \\
\text { desejavam amamentar. Além disso, tinham } \\
\text { incerteza se as crianças consumiam leite } \\
\text { suficiente e se era necessário oferecer leite } \\
\text { artificial. A escala para medir o leite em casa foi } \\
\text { muito importante para as mães, pois com isso } \\
\text { elas sabiam a quantidade ingerida. }\end{array}$ \\
\hline $\begin{array}{c}\text { Slusher T, Slusher IL, Biomdo M, Bode- } \\
\text { Thomas F, Curtis BA, Meier P. Electric } \\
\text { breast pump use increases maternal } \\
\text { milk volume in African nurseries }{ }^{(11)}\end{array}$ & $\begin{array}{l}\text { Comparar a média diária de volume } \\
\text { de leite materno de mães de RNPT, } \\
\text { utilizando: bomba elétrica, não- } \\
\text { elétrica e manual. }\end{array}$ & $\begin{array}{c}\text { ECR. n= } 65 \text { mães de RNPT que eram incapazes de alimentar } \\
\text { diretamente no seio. (amostra aleatória) } \\
\text { G1= expressão de leite por bomba elétrica } \\
\text { G2= expressão de leite por bomba não elétrica por pedal } \\
\text { G3= expressão de leite manual } \\
\text { Variáveis: volume de leite materno, número de dias }\end{array}$ & $\begin{array}{l}\text { O uso das bombas elétricas de mama para medir } \\
\text { a ingesta diária de leite materno mostrou-se } \\
\text { como uma intervenção eficaz nos hospitais para } \\
\text { alcançar o AME nesta população vulnerável. }\end{array}$ \\
\hline $\begin{array}{l}\text { Merewood A, Chamberlain LB, Cook } \\
\text { JT, Philipp BL, Malone K, Bauchner } \mathrm{H} \text {. } \\
\text { The effect of peer counselors on } \\
\text { breastfeeding rates in the neonatal } \\
\text { intensive care unit: results of a } \\
\text { randomized controlled trial }{ }^{(12)}\end{array}$ & $\begin{array}{c}\text { Determinar se os conselheiros em } \\
\text { amamentação tem impacto na } \\
\text { duração do aleitamento materno } \\
\text { de RNPT. }\end{array}$ & $\begin{array}{l}\text { ECR. } \mathrm{n}=108 \text { binômios mãe-filho } \\
\begin{array}{c}\mathrm{GE}=\text { conselheiro em amamentação acompanhou a mãe } \\
\text { semanalmente por } 6 \text { semanas. } \\
\mathrm{GC}=\text { sem conselheiro }\end{array} \\
\text { Variável= presença AM às } 12 \text { semanas após o parto. }\end{array}$ & $\begin{array}{l}\text { As mulheres no GE se sentiam mais culpadas a } \\
\text { respeito da interrupção da amamentação, devido } \\
\text { às relações que desenvolveram com os } \\
\text { conselheiros. No GE aumentou a duração do AM } \\
\text { em } 12 \text { semanas. }\end{array}$ \\
\hline $\begin{array}{c}\text { Pinelli J, Atkinson SA, Saigal S. } \\
\text { Randomized trial of breastfeeding } \\
\text { support in very low-birth-weight } \\
\text { infants }{ }^{(13)}\end{array}$ & $\begin{array}{l}\text { Determinar se o aconselhamento } \\
\text { em amamentação complementar } \\
\text { estruturado em comparação com o } \\
\text { suporte de amamentação } \\
\text { convencional do hospital melhora a } \\
\text { duração da amamentação. }\end{array}$ & $\begin{array}{l}\text { ECR. n=128 RNPT estratificados por peso ao nascer. } \\
\text { O aconselhamento em amamentação complementar estruturado } \\
\text { consistiu em um vídeo sobre AM para RNPT; aconselhamento } \\
\text { individual pelo consultor de lactação; o contato pessoal semanal } \\
\text { no hospital e contato frequente com } 1 \text { ano pós-alta ou até que a } \\
\text { AM tenha sido interrompido. } \\
\text { GE= pais receberam o aconselhamento em amamentação } \\
\text { complementar estruturado ( } n=64 \text { casais) em } 72 \text { horas após o } \\
\text { nascimento. } \\
\text { GC= suporte de amamentação convencional do hospital ( } n=64 \\
\text { casais) } \\
\text { Variáveis: dados demográficos, tipo de aleitamento, importância } \\
\text { da amamentação para os parceiros, experiência de amamentação } \\
\text { na UTIN e experiência de amamentação em casa. }\end{array}$ & $\begin{array}{c}\text { O apoio dos pais prematuros é importante para o } \\
\text { sucesso da amamentação. As mães de ambos os } \\
\text { grupos interromperam a amamentação porque } \\
\text { elas perceberam que não estavam produzindo } \\
\text { leite suficiente. O tabagismo foi um fator } \\
\text { negativo significativo para a manutenção da } \\
\text { amamentação. }\end{array}$ \\
\hline
\end{tabular}




\begin{tabular}{|c|c|c|c|}
\hline Referência & Objetivo & Delineamento & Principais Resultados \\
\hline $\begin{array}{l}\text { Pimenta HP, Moreira ME, Rocha AD, } \\
\text { Gomes JSC, Pinto LW, Lucena SL. } \\
\text { Effects of non-nutritive sucking and } \\
\text { oral stimulation on breastfeeding } \\
\text { rates for preterm, low birth weight } \\
\text { infants: a randomized clinical trial(14) }\end{array}$ & $\begin{array}{l}\text { Determinar a influência da sucção } \\
\text { não-nutritiva e da estimulação oral } \\
\text { nas taxas de amamentação e na alta } \\
\text { hospitalar de RNPT. }\end{array}$ & $\begin{array}{l}\text { ECR. n= } 96 \text { RNPT que permaneceram no estudo até o } 6^{\circ} \text { mês. } \\
\text { GE= participou de um programa de estimulação (sucção não- } \\
\text { nutritiva associado à estimulação sensório-motora-oral), } \\
\text { GC= procedimento simulado a partir do momento em que } \\
\text { atingiam alimentação plena até a alimentação oral completa. } \\
\text { Variáveis: No momento da alta, aos } 3 \text { e aos } 6 \text { meses de idade } \\
\text { corrigida, os RNPT foram classificados como amamentados } \\
\text { (recebendo alimentação através do seio, exclusivo ou não) e não- } \\
\text { amamentados (recebendo alimentação através do seio, exclusivo } \\
\text { ou não), tempo de internação e taxa de amamentação na alta. }\end{array}$ & $\begin{array}{c}\text { A sucção não-nutritiva e a estimulação oral } \\
\text { aumentam a probabilidade do RNPT receber alta } \\
\text { em amamentação, o que pode ser explicado } \\
\text { também pelo menor tempo de internação no } \\
\text { grupo intervenção. }\end{array}$ \\
\hline $\begin{array}{l}\text { Ahmed AH. Breastfeeding preterm } \\
\text { infants: an educational program to } \\
\text { support mothers of preterm infants in } \\
\text { Cairo, Egypt }{ }^{(15)}\end{array}$ & $\begin{array}{l}\text { Desenvolver, implementar e } \\
\text { analisar o efeito de um programa } \\
\text { educativo sobre amamentação de } \\
\text { mães de RNPT. }\end{array}$ & $\begin{array}{c}\text { ECR. n= } 60 \text { mães e seus RNPT (amostra de conveniência). } \\
\text { GE= programa de aleitamento materno com cinco sessões } \\
\text { educacionais durante a hospitalização do bebê e após a alta. } \\
\text { GC= não participaram do programa } \\
\text { Variáveis: conhecimento da amamentação, práticas de } \\
\text { amamentação da mãe, diário de amamentação, perfil da mãe } \\
\text { (idade, escolaridade, ocupação, paridade, história obstétrica, } \\
\text { problemas durante a gravidez, experiência de amamentação e } \\
\text { tipo de parto). }\end{array}$ & $\begin{array}{l}\text { A taxa de AM foi maior no GE, pois o apoio e o } \\
\text { seguimento podem ter aumentado a taxa de AM } \\
\text { na alta. Além disso, cansaço e sonolência durante } \\
\text { a alimentação e sucção fraca foram menos } \\
\text { incidentes no GE. }\end{array}$ \\
\hline $\begin{array}{c}\text { Yildiz A, Arikan D, Gözüm S, Tastekin } \\
\text { A, Budancamana kl. The Effect of the } \\
\text { Odor of Breast Milk on the Time } \\
\text { Needed for Transition From Gavage } \\
\text { to Total Oral Feeding in Preterm } \\
\text { Infants }{ }^{(16)}\end{array}$ & $\begin{array}{l}\text { Investigar o efeito da aplicação do } \\
\text { cheiro do leite materno em RNPT } \\
\text { durante a alimentação por } \\
\text { gavagem no período de transição } \\
\text { para a alimentação oral. }\end{array}$ & $\begin{array}{c}\text { Estudo de caso-controle. } n=80 \text { RNPT } \\
\text { GE= odor de leite materno }(n=40) \\
G C=\text { nenhuma intervenção }(n=40) \\
\text { A intervenção foi realizada diariamente durante três } \\
\text { alimentações até a criança passar para alimentação oral. As } \\
\text { crianças foram pesadas. } \\
\text { Variáveis: peso corporal diário antes da primeira alimentação e } \\
\text { imediatamente antes da alta. }\end{array}$ & $\begin{array}{l}\text { A estimulação com odor do leite materno levou à } \\
\text { maturação precoce do RNPT e da sucção. Além } \\
\text { disso, contribui para a evolução da sucção em } \\
\text { RNPT que leva à tolerância precoce da } \\
\text { alimentação oral com a amamentação. }\end{array}$ \\
\hline $\begin{array}{l}\text { Vianna MN, Barbosa AP, Carvalhaes } \\
\text { AS, Cunha AJ. Music therapy may } \\
\text { increase breastfeeding rates among } \\
\text { mothers of premature newborns: a } \\
\text { randomized controlled Trial }{ }^{(17)}\end{array}$ & $\begin{array}{l}\text { Avaliar o impacto da musicoterapia } \\
\text { no aleitamento materno entre } \\
\text { mães de RNPT. }\end{array}$ & $\begin{array}{c}\text { ECR. } \mathrm{n}=94 \text { mães de RNPT } \\
\text { GE= sessões de musicoterapia } 3 \text { vezes por semana durante } 60 \\
\text { minutos. } \\
\text { GC= atendimento habitual e as consultas de seguimento. } \\
\text { Variáveis: índices de AM na ocasião da alta hospitalar e em } \\
\text { consultas de seguimento (7-15 dias, } 30 \text { e } 60 \text { dias após a alta). }\end{array}$ & $\begin{array}{l}\text { A musicoterapia teve efeito significativo no } \\
\text { aumento do índice de aleitamento materno entre } \\
\text { mães de RNPT. }\end{array}$ \\
\hline
\end{tabular}

Rev. Eletr. Enf. [Internet]. 2013 jan/mar;15(1):253-64. Disponível em: http://dx.doi.org/10.5216/ree.v15i1.17067.

doi: 10.5216/ree.v15i1.17067. 


\begin{tabular}{|c|c|c|c|}
\hline Referência & Objetivo & Delineamento & Principais Resultados \\
\hline $\begin{array}{l}\text { Fewtrel MS, Loh KL, Blake A, Ridout } \\
\text { DA, Hawdon J. Randomised, double } \\
\text { blind trial of oxytocin nasal spray in } \\
\text { mothers expressing breast milk for } \\
\text { preterm infants }{ }^{(18)}\end{array}$ & $\begin{array}{l}\text { Testar a hipótese de que o spray } \\
\text { nasal de ocitocina aumenta a } \\
\text { produção de leite de mães de } \\
\text { RNPT. }\end{array}$ & $\begin{array}{l}\text { ECR duplo-cego. } \mathrm{n}=51 \text { mães de RNPT estratificada por paridade } \\
\text { e idade gestacional (30 semanas e RNPT com mais de } \\
\text { 30semanas). } \\
\text { GE= spray nasal de ocitocina } \\
\text { GC= placebo. } \\
\text { As pulverizações foram antes da expressão de leite com bomba } \\
\text { elétrica em } 5 \text { dias. } \\
\text { Variáveis: volume total de leite ordenhado, padrão de produção } \\
\text { de leite, número de sessões de bombeamento; conteúdo do leite } \\
\text { ordenhado durante um período fixo } 20 \text { minutos no dia 5; a } \\
\text { opinião da mãe ao expressar o leite utilizando o spray. }\end{array}$ & $\begin{array}{c}\text { Muitas mães pararam de expressar leite no início } \\
\text { do período pós-natal porque estão desanimadas } \\
\text { com sua produção de leite, aparentemente baixa. } \\
\text { Não houve diferença significativa, porém a } \\
\text { produção de leite foi ligeiramente superior nas } \\
\text { mães usando spray de ocitocina nos dias 1-3. }\end{array}$ \\
\hline $\begin{array}{l}\text { Rocha NM, Martinez FE, Jorge SM. } \\
\text { Cup or bottle for preterm infants: } \\
\text { effects on oxygen saturation, weight } \\
\text { gain, and breastfeeding }{ }^{(19)}\end{array}$ & $\begin{array}{l}\text { Analisar o impacto do aleitamento } \\
\text { por copo sobre o aleitamento } \\
\text { materno de prematuros. }\end{array}$ & $\begin{array}{l}\text { ECR. n= 83 RNPT estratificados de acordo com peso ao nascer. } \\
\text { GE= copo } \\
\text { GC=mamadeira } \\
\text { Variáveis: método de alimentação, o alimento, o ganho de peso e } \\
\text { as complicações. }\end{array}$ & $\begin{array}{l}\text { Os RNPT alimentados por copo mantiveram o } \\
\text { aleitamento materno por maior tempo do que } \\
\text { aqueles alimentados por mamadeira. }\end{array}$ \\
\hline $\begin{array}{l}\text { Feldman R, Eidelman Al. Direct and } \\
\text { indirect effects of breast milk on the } \\
\text { neurobehavioral and cognitive } \\
\text { development of premature infants }{ }^{(20)}\end{array}$ & $\begin{array}{c}\text { Avaliar o toque materno no } \\
\text { aumento da quantidade de leite } \\
\text { materno ingerido no período } \\
\text { neonatal. }\end{array}$ & $\begin{array}{l}\text { Estudo de coorte. n=86 RNPT classificados pelo consumo de leite } \\
\text { materno: recebem o mínimo (<25\% da nutrição), intermediários } \\
\text { (25-75\%) e substanciais ( }>75 \%) \text { quantidades de leite materno. } \\
\text { Filmou-se dez minutos de interação mãe-bebê. } \\
\text { Variáveis: afeto maternal; toque afetuoso; vocalização materna e } \\
\text { alerta infantil, depressão materna. }\end{array}$ & $\begin{array}{c}\text { As mães que forneceram mais leite tinham toque } \\
\text { carinhoso, conversavam com seus bebês e eles } \\
\text { estavam mais alerta. A depressão materna foi } \\
\text { negativamente relacionada com a quantidade de } \\
\text { leite materno. }\end{array}$ \\
\hline $\begin{array}{l}\text { Ortenstrand A, Winbladh B, } \\
\text { Nordström G, Waldenström U. Early } \\
\text { discharge of preterm infants followed } \\
\text { by domiciliary nursing care: parents' } \\
\text { anxiety, assessment of infant health } \\
\text { and breastfeeding } \\
\text { (21) }\end{array}$ & $\begin{array}{l}\text { Avaliar o efeito da alta precoce de } \\
\text { prematuros, seguido por cuidados } \\
\text { domiciliar sobre ansiedade dos pais, } \\
\text { saúde da criança e aleitamento } \\
\text { materno. }\end{array}$ & $\begin{array}{c}\text { Estudo de caso-controle. n=70 RNPT } \\
\text { GE= alta precoce, com visitas domiciliar ( } n=37 \text { ) } \\
\text { GC= tratamento padrão ( } n=33 \text { ) } \\
\text { Os dados foram coletados por meio do instrumento Inventário } \\
\text { Traço- Estado de Ansiedade e as informações sobre bem-estar } \\
\text { emocional, experiências de saúde da criança e AM foram } \\
\text { coletadas por meio de questionários em três ocasiões: na } \\
\text { internação, na alta hospitalar, após a conclusão do programa de } \\
\text { atendimento domiciliar e depois de } 1 \text { ano, e no GC nos pontos } \\
\text { correspondentes do tempo, que eram durante a hospitalização, } \\
\text { na alta hospitalar e depois de } 1 \text { ano. }\end{array}$ & $\begin{array}{l}\text { Após a conclusão do programa de atendimento } \\
\text { domiciliar todas as mulheres amamentaram. } \\
\text { Diferenças significativas foram observadas na } \\
\text { duração do AM entre os grupos, mas houve uma } \\
\text { tendência de amamentar menos GC quando } \\
\text { comparadas com mães do GE aos } 6 \text { meses. }\end{array}$ \\
\hline
\end{tabular}

Rev. Eletr. Enf. [Internet]. 2013 jan/mar;15(1):253-64. Disponível em: http://dx.doi.org/10.5216/ree.v15i1.17067.

doi: 10.5216/ree.v15i1.17067. 


\begin{tabular}{|c|c|c|c|}
\hline Referência & Objetivo & Delineamento & Principais Resultados \\
\hline $\begin{array}{c}\text { Friedman S, Flidel-Rimon O, Lavie E, } \\
\text { Shinwell ES. The effect of prenatal } \\
\text { consultation with a neonatologist on } \\
\text { human milk feeding in preterm } \\
\text { infants }\end{array}$ & $\begin{array}{l}\text { Estudar o efeito da consulta pré- } \\
\text { natal sobre a incidência e duração } \\
\text { do aleitamento materno em RNPT. }\end{array}$ & $\begin{array}{l}\text { Estudo de caso-controle. n=92 RNPT, pareados por idade, peso } \\
\text { gestacional e multiplicidade } \\
\text { GC= mães haviam recebido consulta de pré-natal, que enfatizou a } \\
\text { importância do AM. } \\
\text { GC= mães que não receberam uma consulta pré-natal, } \\
\text { principalmente devido à escassez de tempo antes do parto. } \\
\text { Variáveis: idade de início e duração de cada tipo de alimentação } \\
\text { (leite materno exclusivo, alimentação parcial ou exclusiva com } \\
\text { fórmula e alimentação com leite humano), dados maternos } \\
\text { (educação, retorno ao trabalho, filiação religiosa). }\end{array}$ & $\begin{array}{l}\text { Durante a internação, os RNPT de mães que } \\
\text { realizaram consulta de pré-natal receberam leite } \\
\text { humano exclusivamente por mais tempo do que } \\
\text { as mães que não realizaram esse } \\
\text { acompanhamento. }\end{array}$ \\
\hline $\begin{array}{l}\text { Mathur NB, Dhingra D. Perceived } \\
\text { breast milk insufficiency in mothers of } \\
\text { neonates hospitalized in neonatal } \\
\text { intensive care unit }{ }^{(23)}\end{array}$ & $\begin{array}{c}\text { Estudar a percepção de leite } \\
\text { insuficiente de mães de RNPT e } \\
\text { avaliar a duração do AME de mães } \\
\text { que perceberam a insuficiência do } \\
\text { leite. }\end{array}$ & $\begin{array}{c}\text { Estudo de coorte. n=307 RNPT e suas mães. } \\
\text { GC= mães que perceberam a insuficiência do leite materno } \\
\text { GC= mães que perceberam adequada produção de leite } \\
\text { As mães foram aconselhadas para amamentar a criança após a } \\
\text { alta e foram acompanhados por três meses, e os bebês avaliados } \\
\text { para peso, comprimento e perímetro cefálico. } \\
\text { Variáveis: percepção da amamentação, tipo amamentação, } \\
\text { administração de água e de alimentos em casa. }\end{array}$ & $\begin{array}{c}\text { A sucção precoce na mama na internação facilita } \\
\text { a amamentação. A prematuridade está associada } \\
\text { com ansiedade e stress com efeito negativo no } \\
\text { consumo de leite. Mães que acreditavam que seu } \\
\text { leite era insuficiente tiveram dificuldades para } \\
\text { manter o aleitamento. }\end{array}$ \\
\hline $\begin{array}{l}\text { Raimbault C, Saliba E, } \\
\text { Porter RH. The effect of the odour of } \\
\text { mother's milk on breastfeeding } \\
\text { behaviour of premature neonates }{ }^{(24)}\end{array}$ & $\begin{array}{l}\text { Avaliar os efeitos da exposição dos } \\
\text { RNPT ao odor do leite materno } \\
\text { sobre a amamentação }\end{array}$ & $\begin{array}{c}\text { Estudo de caso-controle. } n=13 \text { RNPT } \\
\text { GC= odor do leite }(n=7) \\
\text { GC= água ( } n=6)\end{array}$ & $\begin{array}{c}\text { A exposição ao odor do leite antes de uma sessão } \\
\text { de AM tornou a sucção dos RNPT mais eficiente, } \\
\text { preparando-os para obter mais facilmente o leite } \\
\text { do seio de sua mãe. }\end{array}$ \\
\hline $\begin{array}{c}\text { Flacking R, Ewald U, Wallin L. Positive } \\
\text { Effect of Kangaroo Mother Care on } \\
\text { Long-Term Breastfeeding in Very } \\
\text { Preterm Infants }\end{array}$ & $\begin{array}{l}\text { Investigar o Método Mãe Canguru } \\
\text { (MC) e sua associação com a AM de } \\
1 \text { a } 6 \text { meses em mães de RNPT. }\end{array}$ & $\begin{array}{l}\text { Estudo de coorte, longitudinal e prospectivo. n= } 103 \text { RNPT (<32 } \\
\text { semanas de gestação) e n=197 RNPT ( } 32-36 \text { semanas). Dados do } \\
\text { MC (duração do contato pele-a-pele por dia) foram coletados } \\
\text { com os pais. Os dados sobre AM (se a criança era alimentada com } \\
\text { leite materno; o método utilizado para oferecer o leite materno } \\
\text { infantil, apenas na mama ou na mamadeira, ou outro método; e } \\
\text { tipo de amamentação, exclusivo ou parcial) foram obtidos por } \\
\text { entrevistas telefônicas. Dados sobre características infantis, } \\
\text { paridade, idade materna e tipo de parto foram obtidos no } \\
\text { registro da criança. A escolaridade e tabagismo foram obtidas na } \\
\text { pesquisa enviada } 2 \text { meses após a alta. }\end{array}$ & $\begin{array}{l}\text { As mães de RNPT que amamentaram tiveram } \\
\text { mais experiência no MC. }\end{array}$ \\
\hline
\end{tabular}

Rev. Eletr. Enf. [Internet]. 2013 jan/mar;15(1):253-64. Disponível em: http://dx.doi.org/10.5216/ree.v15i1.17067.

doi: $10.5216 /$ ree.v15i1.17067. 


\begin{tabular}{|c|c|c|c|}
\hline Referência & Objetivo & Delineamento & Principais Resultados \\
\hline $\begin{array}{l}\text { Killersreiter B, Grimmer I, Bührer C, } \\
\text { Dudenhausen JW, Obladen M. Early } \\
\text { cessation of breast milk feeding in } \\
\text { very low birth weight infants } \\
\text { (26) }\end{array}$ & $\begin{array}{l}\text { Avaliar a duração da alimentação } \\
\text { com leite materno e analisar os } \\
\text { fatores de risco para interrupção da } \\
\text { amamentação em RNPT. }\end{array}$ & $\begin{array}{l}\text { Estudo de coorte. n=96 RNPT. } \\
\text { Dados obstétricos e perinatais foram registrados a partir de } \\
\text { prontuários das mães. } \\
\text { Variáveis: Dados sócio-demográficos (educação escolar dos pais, } \\
\text { renda familiar e tabagismo), alimentação diária da criança, a data } \\
\text { de visita da mãe ao seu bebê pela primeira vez na UTIN, e as } \\
\text { frequências semanais das visitas dos pais para o bebê. }\end{array}$ & $\begin{array}{l}\text { A baixa autoestima no corpo ocasiona } \\
\text { insegurança na amamentação. Tabagismo na } \\
\text { gravidez e RNPT com <29 semanas de gestação } \\
\text { teve impacto negativo na amamentação. } 0 \text { baixo } \\
\text { grau de escolaridade e idade materna < } 35 \text { anos } \\
\text { indicou menor duração do aleitamento. }\end{array}$ \\
\hline $\begin{array}{l}\text { Zanardo V, Gambina I, Begley C, Litta } \\
\text { P, Cosmi E, Giustardi A, et al. } \\
\text { Psychological distress and early } \\
\text { lactation performance in mothers of } \\
\text { late preterm infants }\end{array}$ & $\begin{array}{l}\text { Analisar a relação entre o } \\
\text { sofrimento psicológico de mães de } \\
\text { RNPT e o desempenho no início da } \\
\text { lactação. }\end{array}$ & $\begin{array}{c}\text { Estudo de caso-controle, prospectivo. } \\
\text { GE= } 42 \text { mães de RNPT } \\
\text { GC= } 42 \text { mães de recém-nascidos a termo } \\
\text { Ambos: pareados por paridade e tipo de parto. } \\
\text { Variáveis: maternas (dados sociodemográficas, doenças } \\
\text { maternas, doenças crônicas, história gestacional, tabagismo, } \\
\text { assistência psicológica e seu plano para amamentar), a idade } \\
\text { gestacional, peso de nascimento, sexo, índice de Apgar e o tipo } \\
\text { de parto. Foi aplicado o Inventário de Traço-Estado de } \\
\text { Ansiedade, a Escala de Edinburgh para Depressão Pós-Parto e a } \\
\text { Medida de Estresse Psicológico em 3-4 dias pós-parto. }\end{array}$ & $\begin{array}{l}\text { Níveis de ansiedade, depressão e estresse de } \\
\text { mães de RNPT foram mais elevados em } \\
\text { comparação às mães de recém-nascidos a termo, } \\
\text { diminuindo a duração do AM. No puerpério, a } \\
\text { angústia psicológica está exacerbada pela } \\
\text { prematuridade, o que prejudica a amamentação. }\end{array}$ \\
\hline $\begin{array}{l}\text { Flacking R, Wallin L, Ewald U. } \\
\text { Perinatal and socioeconomic } \\
\text { determinants of breastfeeding } \\
\text { duration in very preterm infants }{ }^{(28)}\end{array}$ & $\begin{array}{l}\text { Descrever o impacto da } \\
\text { prematuridade, tamanho, doenças } \\
\text { e status social e econômico sobre o } \\
\text { aleitamento materno. }\end{array}$ & $\begin{array}{l}\text { Estudo de coorte prospectivo de base populacional. n= } 225 \text { mães } \\
\text { de RNPT } \\
\text { As mães que amamentaram foram comparadas com aquelas que } \\
\text { não amamentaram. } \\
\text { Variáveis: dados de AM (uso de leite materno, exclusivo ou } \\
\text { parcialmente, e não amamentado); idade da criança ( } 2,4,6,9 \text { e } \\
12 \text { meses); idade gestacional, o peso e o comprimento ao nascer. }\end{array}$ & $\begin{array}{l}\text { As sequelas neonatais constituem uma situação } \\
\text { mais estressante para as mães e interferem na } \\
\text { amamentação. Um baixo nível de escolaridade } \\
\text { materna constitui-se em risco de desmame. }\end{array}$ \\
\hline $\begin{array}{c}\text { Dall'Oglio I, Salvatori G, Bonci E, } \\
\text { Nantini B, D'Agostino G, Dotta A. } \\
\text { Breastfeeding promotion in neonatal } \\
\text { intensive care unit: impact of a new } \\
\text { program toward a BFHI for high-risk } \\
\text { infants }{ }^{(29)}\end{array}$ & $\begin{array}{l}\text { Avaliar o programa de promoção do } \\
\text { aleitamento materno (IHAC) em } \\
\text { UTIN italiana por um período de } \\
\text { tempo. }\end{array}$ &  & $\begin{array}{c}\text { A idade materna permaneceu um fator negativo } \\
\text { para a amamentação. A Iniciativa mostrou que } \\
\text { uma rede complexa de intervenções que são } \\
\text { baseadas em evidências pode ajudar na } \\
\text { manutenção do aleitamento materno. }\end{array}$ \\
\hline
\end{tabular}

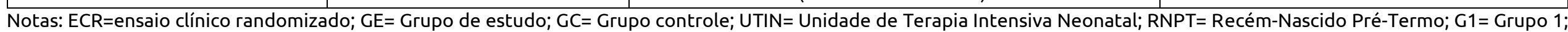

G2= Grupo 2; G3= Grupo 3; AME= Aleitamento Materno Exclusivo; $A M=$ Aleitamento Materno; MC= Método Canguru. 
A análise dos estudos $(n=20)$ possibilitou a identificação de fatores que facilitaram e dificultaram a manutenção do AM dos RNPT.

Dentre os fatores que facilitaram a manutenção da AM destacam-se: a realização da ordenha mamária manual ou elétrica(10-11); o desenvolvimento do vínculo entre a mãe e o bebê(20); apoio dos profissionais de saúde e dos pais para o $A M^{(12-14,21-22)}$; acesso às informações, principalmente às orientações dos profissionais da saúde ${ }^{(10,15,23)}$; o início do AM durante a internação na UTIN e sua associação com o pré-natal(16,22-24); maior tempo no Método Canguru(25); musicoterapia(17) e o uso do copo para oferecer o leite materno ${ }^{(19)}$.

Já dentre os fatores que dificultaram estão: sentimentos de culpa, ansiedade e depressão das mães expressos durante a internação e após a alta ${ }^{(12,18,20-21,23,26-}$ 27); a crença de que seu leite é insuficiente ${ }^{(10,13,23)}$; a oferta de aleitamento artificial(10); o tabagismo ${ }^{(13,26)}$; a prematuridade, a fragilidade do $\mathrm{RN}$ e a dificuldade de sucção no peito materno(15,26); as sequelas neonatais(28); a idade e o grau de escolaridade das mulheres ${ }^{(18,28-29)}$; o tempo de internação e de separação da mãe e filho ${ }^{(14,16,25)}$.

\section{DISCUSSÃO}

A ordenha mamária mostrou-se um fator importante no início e na manutenção da lactação durante a internação do RNPT em uma UTIN. Entretanto, a ordenha mamária pode ser um momento estressante, frustrante e doloroso, que envolve tempo e persistência. A presença de dor ao esgotar pode ser decorrente da técnica incorreta da ordenha mamária, sendo necessárias as orientações dos profissionais de saúde, com vistas a diminuir o desconforto e manter a produção do leite ${ }^{(6)}$.

A respeito da utilização das bombas elétricas para realização da ordenha, destaca-se a importância da informação que as mães recebem dos profissionais de saúde. Assim, é necessário que as mães sejam orientadas e acompanhadas pela equipe de saúde, para que elas possam conhecer e aplicar a técnica correta de utilização das bombas elétricas(6).

O desenvolvimento do vínculo mãe-bebê durante a internação hospitalar facilita a manutenção do AM. O contato estabelecido na amamentação fortalece a ligação entre a mãe e a criança, proporcionando conforto, apego e prazer recíproco ${ }^{(30)}$. A prática do contato precoce pelea-pele auxilia na promoção do AM. Para tanto, valoriza-se a permanência dos pais na UTIN, assim como se estimula o toque e a conversa com o bebê, acarretando efeitos positivos no crescimento e desenvolvimento da criança ${ }^{(30)}$.

O apoio e o estímulo da amamentação pelos profissionais de saúde, especialmente no início do AM, são necessários para auxiliar as mães a manejar os problemas precoces no estabelecimento da AM. Destacase a importância do enfermeiro no processo de amamentação, no sentido de ampliar as estratégias de promoção do $A M$, envolvendo a família, bem como incentivando e apoiando a nutriz ${ }^{(31)}$.

O fato de a mãe receber apoio do marido ou companheiro exerce uma influência positiva na manutenção do AM. Durante o período de amamentação, o pai pode apoiar a mãe auxiliando-a nos afazeres da casa e com os cuidados aos outros filhos(31).

$\mathrm{O}$ acesso às informações, principalmente às orientações dos profissionais de saúde, evidenciou-se como um fator de influência na manutenção do AM de RNPT. A equipe de saúde deve garantir aos pais e familiares orientações apropriadas quanto aos benefícios da amamentação para a mãe, para a criança, para a família e para a sociedade ${ }^{(32)}$.

As informações devem buscar a solução de problemas, no sentido de prevenir e ajudar a mãe a superar as dificuldades que o processo de amamentação pode ocasionar, com vistas a evitar a introdução precoce de alimentação complementar (31). Diante disso, faz-se necessário comunicar-se com a mãe oferecendo-lhe a informação de que ela necessita, no momento adequado, principalmente, quando ela está em condições de absorvê-la e aproveitá-la(32).

Quanto ao início do AM, é comum que os RNPT ao internarem na UTIN não recebam nenhum tipo de alimentação, incluindo o leite materno. O seu início depende da evolução clínica do RN, sendo recomendável que os bebês recebam leite materno 0 mais precocemente possível, por sonda orogástrica (SOG) ou via oral, para atender as necessidades de aporte proteicoenergético e imunológico que ele necessita ${ }^{(33)}$. O início tardio do AM pode comprometer o sucesso da amamentação e aumentar o risco de morte infantil(1).

A implantação do MC representa uma estratégia importante para a sobrevivência do RN, além de tornar a mãe segura e atuante no cuidado de seu filho. O Método 
também assegura a proximidade, afetividade e integralidade no cuidado, propiciando o início e a manutenção do AM. Promove e aumenta as taxas de AME na alta hospitalar, além de adiar a introdução precoce de outros alimentos $^{(1)}$. Esse método envolve a mãe, o pai e a família nos cuidados do RNPT, levando a um atendimento humanizado e de qualidade, que pode reduzir o tempo de internação e a taxa de infecção hospitalar ${ }^{(1)}$.

A utilização do copo, para oferta da alimentação, é uma técnica simples que favorece a amamentação. A técnica de oferta de leite no copo pode ser realizada pelas mães e proporciona aos RNPT maior estabilidade fisiológica e menor impacto no AME no momento da alta hospitalar (33).

O uso do copo fornece uma experiência oral positiva para o $\mathrm{RN}$, pois ele pode controlar o ritmo de sucção e de respiração, sendo que a deglutição ocorre quando o bebê está pronto. Assim, o aleitamento por copo pode contribuir para o estabelecimento da amamentação de sucesso em um curto período(34).

O aleitamento por copo é visto como uma maneira de evitar o uso de bicos artificiais. Por outro lado, os mecanismos orais utilizados em aleitamento por copo, ou a sua segurança e eficácia, são pouco descritos na literatura, demonstrando a falta de evidência a respeito da segurança do método(35).

O estado emocional das mães durante a internação e após a alta hospitalar do seu filho prematuro foi apontado como um fator que pode dificultar a AM dos mesmos. $O$ nascimento prematuro favorece os sentimentos negativos e, muitas vezes, compromete a afetividade entre pais e filhos. Soma-se o fato de não poder pegar o filho no colo, o que se torna frustrante para a mãe. Compreende-se a complexidade das emoções e dos sentimentos vivenciados por ela, quando o nascimento do filho implica na internação em UTIN. O medo do fracasso no aleitamento produz sentimentos negativos nas mães, os quais colaboram para a diminuição da produção de leite ${ }^{(30,36)}$.

Quanto às crenças, especialmente do leite insuficiente, geram sentimentos negativos associados à incapacidade de nutrir o filho, que influenciam na inibição de ocitocina, hormônio responsável pela descida do leite. Pode estar relacionada com a insegurança da nutriz em relação à prática da amamentação(37).

Diante disso, as mães optam pela utilização de fórmulas como a segunda melhor escolha de alimentação e o método mais fácil, com ausência de dor ou desconforto. O aleitamento artificial é mais frequente após a alta hospitalar dos bebês, quando as mães encontram desafios para manutenção do AME no domicílio. Campanhas publicitárias maciças realizadas no passado pela indústria de substitutos do leite materno ainda repercutem na percepção de inocuidade do uso de fórmulas, o que parece gerar uma permissividade e tolerância maior quanto a seu uso ${ }^{(6)}$.

O tabagismo também parece ter uma influência negativa na manutenção do AM dos RNPT. A maioria das mães sabe que a nicotina, presente nos cigarros, passa para o leite, porém elas não conseguem parar de fumar e até interrompem o AM. Nenhuma substância que causa dependência química deve ser ingerida pelas mães lactantes, não só pelos efeitos fisiológicos sobre a criança, mas também pelos danos à saúde física e emocional das mesmas ${ }^{(38)}$.

Dentre os fatores clínicos do RN, a prematuridade e a fragilidade foram apontados como condições desfavoráveis para se estabelecer a amamentação. Características próprias da prematuridade, como a imaturidade clínica, a fraca sucção no seio materno, o cansaço e a sonolência, requerem do RNPT e da mãe maior tempo para estabelecer a nutrição eficiente e adequada. A fragilidade dos RNPT é apontada como contribuinte para a possibilidade eminente de riscos, agravos e sequelas no processo de crescimento e de desenvolvimento de diversos tipos como atraso motor, transtorno do desenvolvimento da coordenação, hiperatividade, retinopatia, displasia broncopulmonar ${ }^{(39)}$.

As sequelas neonatais podem ser decorrentes de alguns problemas como: síndrome do desconforto respiratório, apneia, displasia broncopulmonar, persistência do canal arterial, termorregulação ineficaz, hipoglicemia, hemorragia intraventricular, disfunção gastrointestinal, retinopatia, hiperbilirrubinemia e infecção. As sequelas podem dificultar o início e a manutenção do AM, prejudicando o desenvolvimento do $\mathrm{RNPT}^{(40)}$.

A idade materna está relacionada a menor duração do AME. Especialmente quando estão na adolescência, a insegurança e a falta de confiança em si mesmas, próprias da idade, as fazem desmamar seus filhos. As mães de menor grau de escolaridade iniciam o pré-natal 
tardiamente, bem como adiam a decisão da forma de alimentação do filho(41).

Quanto maior o tempo de internação, menor a probabilidade de manter o AME. O período de internação do RN na UTIN torna-se um obstáculo para a formação de vínculo e apego entre mãe e bebê(30), dificultando o início e a manutenção do AM. A equipe deve ser empática e sensível com as mães e familiares, para que eles possam enfrentar a internação do bebê da melhor maneira, minimizando os danos e prejuízos que esse período possa acarretar no $\mathrm{AM}^{(31)}$.

Constatou-se que a separação da mãe e filho durante a internação é um fator que interfere no aleitamento. Além disso, o ambiente complexo da UTIN, com normas e rotinas rígidas, resultam na separação do binômio mãefilho, enfraquecendo o vínculo mãe-filho e dificultando à manutenção da amamentação(42).

\section{CONCLUSÕES}

A avaliação das evidências sobre os fatores associados à manutenção do AM de RNPT apontou fatores facilitadores e dificultadores, auxiliando os profissionais de saúde a melhorarem a sua prática no sentido de promover, proteger e apoiar a prática do AM desde o parto prematuro, durante a internação na UTI e no preparo para alta hospitalar.

Рara potencializar os fatores facilitadores, recomenda-se o desenvolvimento de ações educativas para o estabelecimento da ordenha e o início precoce, de acordo com as condições clínicas do RNPT. Faz-se também importante promover estratégias para o desenvolvimento do vínculo mãe-bebê, o que inclui o $M C$, bem como o acolhimento da família, considerando a importância de se ter uma rede de familiares, profissionais e instituições para atendê-los de maneira integral e humanizada.

Рага minimizar os fatores dificultadores, os profissionais de saúde devem ter especial atenção às mães que manifestam sentimentos e crenças que interferem negativamente no $A M$, assim como a prescrição do aleitamento artificial como complemento e o uso de dispositivos tecnológicos. Soma-se a necessidade de ações específicas a mães adolescentes, com baixa escolaridade e renda, e também aquelas que têm maior tempo de internação.

São ainda necessárias ações multiprofissionais para manutenção e promoção da estabilidade clínica dos RNPT, estimulando a sucção não-nutritiva para o início precoce e manutenção do AME.

Retoma-se o compromisso de promover, proteger e apoiar o AM, contribuindo para o aumento dos índices de AME até seis meses. Diante disso, faz-se necessário investir em pesquisas com delineamentos que apresentem evidências fortes relativas ao tema investigado, especialmente na prática de saúde.

8. Mendes KDS, Silveira RCCP, Galvão CM. Revisão integrativa: método de pesquisa para a incorporação de evidências na saúde e na enfermagem. Texto Contexto Enferm. 2008;17(4):758-64.

9. Melnyk BM, Fineout-Overholt E. Making the case for evidencebased practice. In: Melnyk BM, Fineout-Overholt E. Evidencebased practice in nursing \& healthcare. A guide to best practice. Philadelphia: Lippincot Williams \& Wilkins; 2005. p. 3-24.

10. Hurst NM, Meier PP, Engstrom JL, Myatt A. Mothers performing in-home measurement of milk intake during breastfeeding of their preterm infants: maternal reactions and feeding outcomes. J HumLact.2004;20(2):178-87.

11. Slusher T, Slusher IL, Biomdo M, Bode-Thomas F, Curtis BA, Meier P. Electric breast pump use increases maternal milk volume in African nurseries. J Trop Pediatr. 2007; 53(2):125-30. 12. Merewood A, Chamberlain LB, Cook JT, Philipp BL, Malone K, Bauchner $H$. The effect of peer counselors on breastfeeding rates in the neonatal intensive care unit: results of a randomized controlled trial. Arch Pediatr Adolesc Med. 2006;160(7):681-5.

13. Pinelli J, Atkinson SA, Saigal S. Randomized trial of breastfeeding support in very low-birth-weight infants. Arch Pediatr Adolesc Med.2001;155(5):548-53.

14. Pimenta HP, Moreira ME, Rocha AD, Gomes Jr SC, Pinto LW, Lucena SL. Effects of non-nutritive sucking and oral stimulation

cuidado neonatal de mulheres participantes de um programa Mãe-Canguru. Interação em Psicologia. 2008;12(2):255-266. 
on breastfeeding rates for preterm, low birth weight infants: a randomized clinical trial. J Pediatr. 2008;84(5):423-7.

15. Ahmed AH. Breastfeeding preterm infants: an educational program to support mothers of preterm infants in Cairo, Egypt. Pediatr Nurs. 2008;34(2):125-30

16. Yildiz A; Arikan D; Gözüm S; Tastekin A; Budancamanak I. The Effect of the Odor of Breast Milk on the Time Needed for Transition From Gavage to Total Oral Feeding in Preterm Infants. J Nurs Scholarsh; 2011;43(3):265-73.

17. Vianna MN; Barbosa AP; Carvalhaes AS; Cunha AJ. Music therapy may increase breastfeeding rates among mothers of premature newborns: a randomized controlled Trial. J Pediatr. 2011;87(3):206-12.

18. Fewtrell MS, Loh $K L$, Blake $A$, Ridout $D A$, Hawdon J. Randomised, double blind trial of oxytocin nasal spray in mothers expressing breast milk for preterm infants. Arch Dis Child Fetal Neonatal Ed. 2006;91(3):169-74.

19. Rocha NM, Martinez FE, Jorge SM. Cup or bottle for preterm infants: effects on oxygen saturation, weight gain, and breastfeeding. J Hum Lact. 2002;18(2):132-8.

20. Feldman R, Eidelman Al. Direct and indirect effects of breast milk on the neurobehavioral and cognitive development of premature infants. Dev Psychobiol. 2003;43(2):109-19.

21. Ortenstrand A, Winbladh B, Nordström G, Waldenström U. Early discharge of preterm infants followed by domiciliary nursing care: parents' anxiety, assessment of infant health and breastfeeding. Acta Paediatr. 2001;90(10):1190-5.

22. Friedman S, Flidel-Rimon O, Lavie E, Shinwell ES. The effect of prenatal consultation with a neonatologist on human milk feeding in preterm infants. Acta Paediatr. 2004; 93(6):775-8.

23. Mathur NB, Dhingra D. Perceived breast milk insufficiency in mothers of neonates hospitalized in neonatal intensive care unit. Indian J Pediatr. 2009;76(10):1003-6.

24. Raimbault $C$, Saliba E, Porter RH. The effect of the odour of mother's milk on breastfeeding behaviour of premature neonates. Acta Paediatr. 2007;96(3):368-71

25. Flacking R; Ewald U; Wallin L. Positive Effect of Kangaroo Mother Care on Long-Term Breastfeeding in Very Preterm Infants. J Obstet Gynecol Neonatal Nurs 2011;40(2):190-7.

26. Killersreiter B, Grimmer I, Bührer C, Dudenhausen JW, Obladen M. Early cessation of breast milk feeding in very low birth weight infants. Early Hum Dev.2001; 60(3):193-205.

27. Zanardo V; Gambina I; Begley C; Litta P; Cosmi E; Giustardi A; et al. Psychological distress and early lactation performance in mothers of late preterm infants. Early Hum Dev. 2011;87(4):3213.

28. Flacking R, Wallin L, Ewald U. Perinatal and socioeconomic determinants of breastfeeding duration in very preterm infants. Acta Paediatr. 2007;96(8):1126-30.

29. Dall'Oglio I, Salvatori G, Bonci E, Nantini B, D'Agostino G, Dotta $A$. Breastfeeding promotion in neonatal intensive care unit: impact of a new program toward a BFHI for high-risk infants. Acta Paediatr.2007;96(11):1626-31.

30. Cruz ARM, Oliveira MMC, Cardoso MVLML, Lúcio IML. Sentimentos e expectativas da mãe com filho prematuro em ventilação mecânica. Rev. Eletr. Enf. 2010;12(1):133-9.

31. Bullon RB, Cardoso FA, Peixoto HM, Miranda LF. A influência da família e o papel do enfermeiro na promoção do aleitamento materno. Universitas: Ciências da Saúde. 2009;7(2):49-70.

32. Silva RQ, Gubert MB. Qualidade das informações sobre aleitamento materno e alimentação complementar em sites brasileiros de profissionais de saúde disponíveis na internet. Rev. Bras. Saúde Matern. Infant. 2010;10(3):331-40.

33. Mendonça LBA, Menezes MM, Rolim KMC, Lima FET. Cuidados ao recém-nascido prematuro em uso de sonda orogástrica: conhecimento da equipe de enfermagem. Rev. Rene. 2010;11(Número Especial):178-85.
34. Gupta A, Khanna K, Chattree S. Cup feeding: an alternative to bottle feeding in a neonatal intensive care unit. $J$ Trop Pediatr. 1999;45(2):108-10.

35. Dowling DA, Meier PP, DiFiore JM, Blatz M, Martin RJ. Cupfeeding for preterm infants: mechanics and safety. J Hum Lact. 2002;18(1):13-20.

36. Marques DM, Pereira AL. Amamentar: sempre benefícios, nem sempre prazer. Cienc Cuid Saúde. 2010;9(2):214-19.

37. Simiquel FL, Marcello JF, Taucci RA, Cachaldora TN. Aleitamento materno: principais dificuldades da lactante e do lactente e levantamento sobre o conhecimento dos seus benefícios em relação à fonoaudiologia. CES Revista - Juiz de Fora 2006;20(1):221-36.

38. Machado JB, Lopes MHI. Abordagem do tabagismo na gestação. Scientia Medica. 2009;19(2):75-80.

39. Ramos HAC, Cuman RKN. Fatores de risco para prematuridade: pesquisa documental. Esc Anna Nery Rev Enferm. 2009 abr-jun; 13(2):297-304.

40. Lara $P$, Sucena LP, Furlan MFFM. Incidência da utilização de leite materno ordenhado em uma Unidade de Terapia Intensiva neonatal e caracterização dos recém-nascidos. Arq Ciênc Saúde 2008; 15(2):82-9.

41. Henry BA, Nicolau AIO, Américo CF, Ximenes LB, Bernheim RG, Oriá MOB. Socio-Cultural factors influencing breastfeeding practices among low-income women in Fortaleza-Ceará-Brazil: a Leininger's Sunrise Model Perspective. Rev. Enfermería Global. 2010;19.

42. Dittz ES, Sena RR, Motta JAC, Duarte ED. Cuidado materno ao recém-nascido na Unidade de Terapia Intensiva Neonatal: possibilidades e desafios. Ciencia y Enfermeria 2011;17(1):45-55.

Artigo recebido em 02/02/2011.

Aprovado para publicação em 12/04/2012.

Artigo publicado em 31/03/2013. 